Revue d'histoire de l'Amérique française

REVUE D.HISTOIRE DE L'AMÉRIQUE FRANÇAISE

\title{
Et si Paul Sauvé n’avait jamais prononcé le « Désormais... » ?
}

\section{Alexandre Turgeon}

Volume 67, numéro 1, été 2013

URI : https://id.erudit.org/iderudit/1026615ar

DOI : https://doi.org/10.7202/1026615ar

Aller au sommaire du numéro

\section{Éditeur(s)}

Institut d'histoire de l'Amérique française

\section{ISSN}

0035-2357 (imprimé)

1492-1383 (numérique)

Découvrir la revue

\section{Citer cet article}

Turgeon, A. (2013). Et si Paul Sauvé n’avait jamais prononcé le " Désormais... » ? Revue d'histoire de l'Amérique française, 67(1), 33-56. https://doi.org/10.7202/1026615ar

\section{Résumé de l'article}

" Désormais... ». Il n’y aurait pas de mots plus célèbres dans l'histoire du Québec. Le premier ministre Paul Sauvé l'aurait prononcé sans cesse à l'automne 1959, alors qu'il succède à Maurice Duplessis, afin de tirer un trait définitif entre deux mondes : la Grande Noirceur duplessiste et la Révolution tranquille. Que Paul Sauvé ait prononcé le « Désormais... ", on ne saurait en douter. Les contemporains en attestent, maints historiens le confirment. Osons malgré tout une question : Et si Paul Sauvé n'avait jamais prononcé le " Désormais... » ? C'est ce qui apparaît au terme de recherches exhaustives menées dans les journaux et documents de l'époque. Cet article propose d'étudier comment le " Désormais... » est devenu un mythistoire. 


\title{
Et si Paul Sauvé n'avait jamais prononcé le “Désormais...”?
}

\author{
Alexandre Turgeon ${ }^{\mathrm{I}}$ \\ Département des sciences historiques \\ Université Laval
}

RÉSUMÉ • «Désormais... ». II n'y aurait pas de mots plus célèbres dans l'histoire du Québec. Le premier ministre Paul Sauvé l'aurait prononcé sans cesse à l'automne 1959, alors qu'il succède à Maurice Duplessis, afin de tirer un trait définitif entre deux mondes: la Grande Noirceur duplessiste et la Révolution tranquille. Que Paul Sauvé ait prononcé le «Désormais... », on ne saurait en douter. Les contemporains en attestent, maints historiens le confirment. Osons malgré tout une question: Et si Paul Sauvé n'avait jamais prononcé le «Désormais... »? C'est ce qui apparaît au terme de recherches exhaustives menées dans les journaux et documents de l'époque. Cet article propose d'étudier comment le «Désormais... » est devenu un mythistoire.

ABstract • "Désormais..." (From Now On). There might not be a more famous word in Québec history. As he was succeeding Maurice Duplessis as Premier during the autumn of 1959, Paul Sauvé is said to have used this word repeatedly to draw a line between two worlds: the Great Darkness of the Duplessis era and the Quiet Revolution. There can be no doubt that Paul Sauvé routinely used the word "Désormais...", as several contemporaries and historians have confirmed. Nonetheless, we ask the question: What if Paul Sauvé had never uttered "Désormais..."? Through an exhaustive research in the archives and newspapers of that era, this paper examines how the word "Désormais..." became a mythistory.

1. L'auteur tient à remercier les évaluateurs anonymes ainsi que Christian Blais, Anne Trépanier, Jocelyn Létourneau, Maxime Morin et en particulier Raphaël Gani pour leurs conseils et commentaires. La rédaction de cet article a été rendue possible grâce à l'appui financier du Fonds de recherche du Québec - Société et culture. 
De septembre à janvier, Paul Sauvé, débordé, n'utilisa plus dans ses discours qu'un seul mot: désormais. Avec ce vocable, il séparait deux modes de vie politique, deux ères dont la dernière serait trop brève pour porter son nom.

Georges-Émile Lapalme, témoin, $1970^{2}$

"Désormais": ce mot emblématique de Paul Sauvé, brièvement premier ministre après la mort de Maurice Duplessis, a eu l'effet d'un verrou qui saute.

LuCia FerRetTi, historienne, $1999^{3}$

Il ne sera en poste que 100 jours avant de mourir subitement, mais son célèbre "Désormais", était annonciateur d’un virage majeur. Il a été le premier à ouvrir les vannes du changement.

Jean Charest, premier ministre du Québec, $2010^{4}$

L

es auteurs de ces citations sont des personnages de qualité de la société québécoise. Le premier fut chef du Parti libéral du Québec entre 1950 et 1958 et est considéré comme l'un des précurseurs de la Révolution tranquille $e^{5}$. La deuxième est une historienne chevronnée, spécialiste de l'histoire socioreligieuse du Québec, dont l'œuvre a été récompensée par le Prix Gérard-Parizeau 2011. Le troisième, enfin, a été premier ministre du Québec entre 2003 et 2012; c'est à ce titre qu'il fait cette déclaration dans le cadre d'une entreprise commémorative célébrant les cinquante ans de la Révolution tranquille. Ces trois citations relèvent chacune de différentes perspectives: celle du témoin qui puise dans ses souvenirs, celle du chercheur qui étudie un phénomène donné et celle du responsable politique qui présente une politique. Tous trois témoignent à divers degrés d'une seule et même réalité, d'un mot prononcé à profusion par Paul Sauvé: "Désormais...».

Il n'y a peut-être pas de mot plus célèbre dans les arcanes de la mémoire collective ou dans les annales de l'histoire du Québec. Un mot qui s'est

2. Georges-Émile Lapalme, Mémoires. Tome 2: Le vent de l'oubli (Montréal, Leméac, 1970), 277.

3. Lucia Ferretti, Brève histoire de l'Église catholique au Québec (Montréal, Boréal, 1999), 156.

4. Allocution de Jean Charest, le 23 septembre 2010, dans «Paul Sauvé», Révolution tranquille - 50 ans - Un courant d'inspiration, www.revolutiontranquille.gouv.qc.ca [consulté le 15 août 2013].

5. Jean-François Léonard, dir., Georges-Émile Lapalme (Sillery, Presses de l’Université du Québec, 1988), 297 p. ; Jean-Charles Panneton, Georges-Émile Lapalme, précurseur de la Révolution tranquille (Montréal, VLB éditeur, 2000), 190 p. 
plus récemment retrouvé sous les projecteurs lors de la victoire du Parti Québécois, le 4 septembre $2012^{6}$. Ce mot, le premier ministre Paul Sauvé ne cesse de le prononcer à l'automne 1959, alors qu'il succède à Maurice Duplessis, décédé à Schefferville. Par ce mot, répété à toutes les occasions, Paul Sauvé tire un trait définitif entre deux temps et deux mondes: entre la Grande Noirceur duplessiste et la Révolution tranquille, entre l'Ancien et le Nouveau Régime québécois pour le dire avec Léon Dion?

À l'époque comme de nos jours, les contemporains ${ }^{8}$ attestent que Paul Sauvé a prononcé le «Désormais... » et de nombreux chercheurs le confirment depuis cinquante ans ${ }^{9}$. Outre ces témoignages, Paul Sauvé a aussi

6. "Le retour au "nous", Pauline Marois le voyait, raconte-t-on dans son entourage, comme un véritable "désormais". Après la mort de Duplessis, Paul Sauvé, lorsqu'il prend la place du "cheuf”, prononce ce mot célèbre, marquant le début de la Révolution tranquille. C'est aussi à une rupture que la nouvelle chef péquiste voulait convier les nationalistes, le 29 août, lors de son discours d'assemblée de nomination. » Antoine Robitaille, «Le "nous”, c'est lui. Pauline Marois s'est largement inspirée du sociologue Jacques Beauchemin», Le Devoir, 24 septembre 2007, A1.

7. Léon Dion, «De l'ancien... au nouveau régime», Cité libre, XII, 39 (juin-juillet 1961): 3-14.

8. Parmi les plus connus, mentionnons Lionel Bertrand, Quarante ans de souvenirs politiques (SainteThérèse, Lionel Bertrand, 1976), 79; G.-É. Lapalme, Mémoires..., op. cit., 277-278; Gérard Pelletier, Souvenirs. Tome 2: Le temps des choix, 1960-1968 (Montréal, Stanké, 1986), 34-36; Jean-Louis Gagnon, Les apostasies. Tome 2: Les dangers de la vertu (Montréal, La Presse, 1988), 16; Paul Gérin-Lajoie, Combats d'un révolutionnaire tranquille (Montréal, Centre éducatif et culturel, 1989), 173; Léon Dion, Québec 1945-2000. Tome 2: Les intellectuels et le temps de Duplessis (Québec, Les Presses de l'Université Laval, 1993), 16 et 142; Robert Prévost, "Le "désormais" de Paul Sauvé», Histoire Québec, 5,2 (novembre 1999): 21-28; Claude Castonguay, Mémoires d'un révolutionnaire tranquille (Montréal, Boréal, 2005), 38; Roch Bolduc, Le Mandarin de l'ombre. De la Grande Noirceur à la Révolution tranquille (Québec, Septentrion, 2012), 101.

9. Parmi les plus notables, mentionnons Hubert Guindon, «La modernisation du Québec et la légitimité de l'État canadien ", Recherches sociographiques, 18,3 (1977): 339; Denis Monière, Le développement des idéologies au Québec des origines à nos jours (Montréal, Québec/Amérique, 1977), 307; Dorval Brunelle, La désillusion tranquille (Montréal, Hurtubise HMH, 1978), 137; Richard Jones, Duplessis et le gouvernement de l'Union nationale, Brochure de la Société historique du Canada, 35 (1983), 19; Dale C. Thomson, Jean Lesage \& the Quiet Revolution (Toronto, Macmillan of Canada, 1984), 78 et 81; Jean Hamelin, Histoire du catholicisme québécois. Volume 3: Le XX siècle. Tome 2: De 1940 à nos jours (Montréal, Boréal Express, 1984), 238; Paul-André Linteau, René Durocher, Jean-Claude Robert et François Ricard, Histoire du Québec contemporain. Tome 2: Le Québec depuis 1930 (Montréal, Boréal, 1986), 340, 598 et 612; Réjean Pelletier, Partis politiques et société québécoise : de Duplessis à Bourassa 1944-1970 (Montréal, Québec/Amérique, 1989), 238; Jocelyn Létourneau, "La saga du Québec moderne en images», Genèses: Sciences sociales et histoire, 4,1 (mai 1991): 63 ; Jacques Mathieu et Jacques Lacoursière, Les mémoires québécoises (Québec, Les Presses de l'Université Laval, 1991), 26; Guy Rocher, «Beyond the Quiet Revolution», dans John Meisel, Guy Rocher et Arthur Silver, dir., As I recall/Si je me souviens bien: historical perspectives (Montréal, Institut de recherche en politiques publiques, 1999), 204; L. Ferretti, Brève histoire..., op. cit., 156; Michel SarraBournet, «Duplessis, Maurice Le Noblet», Dictionnaire biographique du Canada en ligne, www.biographi. $\mathrm{ca} / \mathrm{fr} /$ [consulté le 15 août 2013]; Yvan Lamonde, «Malaise dans la culture québécoise: les méprises à propos de la Révolution tranquille», dans Guy Berthiaume et Claude Corbo, dir., La Révolution tranquille en héritage (Montréal, Boréal, 2011), 13 ; Éric Bédard, L'Histoire du Québec pour les Nuls (Paris, Éditions First-Gründ, 2012), 234-235. 
fait l'objet d'expositions ${ }^{10}$, d'un documentaire ${ }^{11}$ et le gouvernement du Québec l'a récemment honoré comme Grand Artisan de la Révolution tranquille, rappelant à cette occasion son mot vénérable. De toute évidence, devant ces témoignages, devant ces sources qui s'accumulent, le «Désormais...» est un fait historique, avéré. On ne saurait en douter. Osons malgré tout une question: Et si Paul Sauvé n’avait jamais prononcé le «Désormais... »? Malgré les apparences, c’est la conclusion qui s’impose au terme de recherches exhaustives menées dans les journaux et documents de l'époque, sur lesquelles nous reviendrons. C'est donc dire qu'il s'agit d'un mythistoire.

Qu'entendons-nous par «mythistoire»? Formé des mots «mythe» et "histoire», "mythistoire» est toutefois plus proche du premier que du second. «Mythe» et "mythistoire» sont deux concepts qui ont été popularisés au Québec respectivement par Gérard Bouchard et Jocelyn Létourneau. Dans un cas comme dans l'autre, il s'agit de la représentation d'un objet donné qui relève, d'une part, du domaine de la fiction, du folklore, des légendes, mais qui s'inscrit, s'enracine, d'autre part, dans le tangible, l'avéré, l'empirique. Pour Bouchard, le mythe est

une instauration durable de valeur et de sens (entretenant ou non un rapport avec le sacré) qui commande d'autres valeurs et d'autres sens, qui fixe des repères à l'identité et une direction à l'action collective, qui s'incarne, enfin, aussi bien dans des objets et des personnages que dans des événements et des récits ${ }^{12}$.

Tandis que pour Létourneau, le mythistoire est

tout à la fois, une fiction réaliste, un système d'explication et un message mobilisateur qui rencontrent une demande de sens, si ce n'est un désir de croyance, chez ses destinataires. Ce qui explique la force et assure la persistance du mythistoire tient à l'arrimage qui existe entre la structure représentative et la matière représentée ${ }^{13}$.

Comme on peut le voir, les définitions de ces deux concepts se recoupent, renvoient au même phénomène. C'est afin de les distinguer l'un de l'autre que nous faisons appel aux catégories de Carlo Ginzburg qui s’in-

10. Paul Labonne, Paul Sauvé. Désormais, l'avenir (1907-1960) (Outremont, Point de fuite, 2003), 109 p.

11. Paul Carvalho et Paul Labonne, Paul Sauvé: Désormais l'avenir! (Montréal, Productions Films Perception, 2008), 60 minutes.

12. Gérard Bouchard, «L’imaginaire de la grande noirceur et de la révolution tranquille: fictions identitaires et jeux de mémoire au Québec», Recherches sociographiques, XLVI, 3 (septembre-décembre 2005): 413 .

13. Jocelyn Létourneau, "Mythistoires de Losers: introduction au roman historial des Québécois d'héritage canadien-français ", Histoire sociale/Social History, 39,7 (mai/May 2006) : 162. 
téresse aux rapports entre le vrai, le fictif et le faux, soit «le fictif qui se fait passer pour vrai ${ }^{14} »$. Ces rapports, nous nous proposons de les articuler selon deux modes: soit le mythe - où prime le fictif - et le mythistoire - où prime le faux. Ce qui les différencie, c'est la place qui est donnée au fictif, ou plutôt la place prise par ce dernier dans les rapports avec le vrai. $S$ 'il est admis qu'une telle représentation concerne à la fois le réel et la fiction, le vrai et le fictif, dont les dosages peuvent varier: nous sommes alors en face d'un mythe. Les frontières entre le vrai et le fictif sont toutefois poreuses, et le rapport entre ces deux termes bouleversé - ou plutôt dissipé, dissous - du moment que le fictif se fait passer pour vrai: c'est ce que nous appelons mythistoire.

Ainsi donc, le «Désormais...» de Paul Sauvé est un mythistoire. Pour reprendre les termes de Carlo Ginzburg, c'est un faux, c'est-à-dire que le fictif passe pour vrai. Au-delà de la simple correction des souvenirs et de la banale rectification des faits historiques, quelles sont les implications de cette situation, de cet état de fait? Pour le dire autrement, il ne s'agit pas seulement de déterminer que le «Désormais...» de Paul Sauvé est un mythistoire, mais de montrer pourquoi et comment il en fut ainsi. Tels sont les objectifs de cet article.

Pour ce faire, nous commencerons par établir la nature du «Désormais...", dans la littérature comme dans les imaginaires, afin de bien montrer l'importance du phénomène. Nous reviendrons ensuite sur les "cent jours» de Paul Sauvé, où nous ferons la démonstration que le premier ministre n'a jamais prononcé le «Désormais...", qu'il s'agit bien d’un mythistoire. Pour finir, notre attention se portera sur sa genèse. À cette occasion, c'est le travail de l'éditorialiste André Laurendeau, du journal Le Devoir, qui ressort, lui qui est le premier à mettre ce mot à la bouche de Paul Sauvé.

\section{ENTRE LA GRANDE NOIRCEUR DUPLESSISTE ET LA RÉVOLUTION TRANQUILLE}

D’emblée, une première question se pose: qu’est-ce que le «Désormais...»? Il peut ainsi s'agir, selon les auteurs qui ont redoublé d'imagination et rivalisé d'originalité, d'un "surnom ${ }^{15} »$, d'une «formule ${ }^{16}$ », d'un «leit-

14. Carlo Ginzburg, Le fil et les traces. Vrai faux fictif (Lagrasse, Verdier, 2010), 16.

15. André D’Allemagne, Le colonialisme au Québec : essai (Montréal, Éditions R-B, 1966), 15; Robert Lacour-Gayet, Histoire du Canada (Paris, Fayard, 1966), 538.

16. Jean Hamelin et André Garon, «Introduction: La vie politique au Québec de 1956 à 1966 » dans Vincent Lemieux, dir., Quatre élections provinciales au Québec, 1956-1966 (Québec, Les Presses de l’Université Laval, 1969), 12 ; J. Létourneau, «La saga...», loc. cit., 63. 
motiv $^{17}$ », d'une "politique ${ }^{18}$ », d'un «motto ${ }^{19}$ ", ou encore d'un «slogan ${ }^{20} »$, le terme qui revient de loin le plus souvent dans la littérature. En tout premier lieu, l'adverbe «désormais» est un marqueur de rupture temporelle, en cela qu'il distingue très nettement un avant d'un après qui sera tout autre. D'autres termes remplissent également cette fonction: pensons à «maintenant», «à l'avenir » et en particulier à «dorénavant». À la différence de "dorénavant», notent Francis Chatreauraynaud et Marianne Doury, «désormais» peut toutefois être utilisé pour qualifier un objet ou pour clore une phrase ${ }^{21}$.

Dans le contexte québécois, le «Désormais...» de Paul Sauvé trace une ligne de démarcation nette entre la Grande Noirceur duplessiste, d'une part, et la Révolution tranquille, d'autre part, où il s'insère dans une certaine trame narrative. L'extrait suivant en est un bon exemple:

Après sa nomination comme successeur de Maurice Duplessis, [Paul Sauvé] indique rapidement que les choses vont changer en prononçant un simple mot, devenu célèbre partout au Québec, «désormais». Le règne de 100 jours de Sauvé marque le début de la Révolution tranquille, parce qu'il provoque une renaissance et permet de régler plusieurs questions en suspens, notamment celles de l'assurance-maladie et des subventions aux universités ${ }^{22}$.

Cet extrait réunit tous les éléments de l'intrigue, marquée par son inachèvement, si ce n'est son interruption. Reprenons chacun des éléments de l'intrigue.

L'élément déclencheur est le décès de Maurice Duplessis, emporté par une hémorragie cérébrale le 7 septembre 1959, à Schefferville. Appelé à lui succéder, Paul Sauvé se fait le porte-parole du changement en utilisant un terme à profusion, "Désormais...", qui marque tout autant la fin de la Grande Noirceur duplessiste - avec la mort de Duplessis - que le début

17. René Lévesque, La passion du Québec (Montréal, Québec/Amérique, 1978), 234; J. Mathieu et J. Lacoursière, Les mémoires québécoises..., op. cit., 26.

18. Gérard Bergeron, Du duplessisme à Trudeau et Bourassa, 1956-1971 (Montréal, Parti pris, 1971), 147; Gérard Bergeron, Pratique de l'État au Québec (Montréal, Québec/Amérique, 1984), 68.

19. Renate Usmiani, Michel Tremblay (Vancouver, Douglas \& McIntyre, 1982), 12 ; Marguerite Paulin, Maurice Duplessis : Powerbroker, politician (Montréal, XYZ Pub, 2005), 207.

20. D. Monière, Le développement des idéologies..., op. cit., 307; J. Hamelin, Histoire du catholicisme québécois..., op. cit., 238 ; P.-A. Linteau, R. Durocher, J.-C. Robert et F. Ricard, Histoire du Québec contemporain..., op. cit., 340; M. Sarra-Bournet, "Duplessis...», loc. cit.

21. Pensons seulement à la formule «le désormais célèbre», par exemple. Francis Chateauraynaud et Marianne Doury, “"Désormais...” Essai sur les fonctions argumentatives d’un marqueur de rupture temporelle ", Argumentation et Analyse du Discours, 4 (2010), aad.revues.org/ [consulté le 5 septembre 2013].

22. Daniel Latouche, «Joseph-Mignault-Paul Sauvé», L'Encyclopédie canadienne, www.thecanadianencyclopedia.com [consulté le 4 septembre 2013]. 
de la Révolution tranquille - avec les nombreuses réformes que Sauvé a tout juste le temps de mettre de l'avant. Mais Paul Sauvé n'est premier ministre que durant 114 jours, appelés les «cent jours», avant de mourir à son tour dans l'exercice de ses fonctions. Il laisse ainsi une œuvre inachevée, interrompue. Ce sera à Jean Lesage et à son "équipe du tonnerre » de la continuer, de faire la Révolution tranquille, après leur victoire du 22 juin 1960.

L'intrigue ne se retrouve pas toujours telle quelle, avec tous ses éléments. C'est le cas notamment du lieu ou encore du moment où Paul Sauvé aurait prononcé le «Désormais... ». Il l'aurait ainsi employé dans ses discours ${ }^{23}$, dans sa première conférence de presse ${ }^{24}$, dans son discours inaugural ${ }^{25}$, au premier caucus de son parti ${ }^{26}$, s'il ne l'a pas fait à l'Assemblée législative ${ }^{27}$ ou encore à l'Université de Montréal ${ }^{28}$. Ils sont nombreux du reste à souligner que Paul Sauvé débute ses discours par cet adverbe ${ }^{29}$ - certains iront même jusqu'à soutenir qu'il l'emploie dans chacun de ses discours $^{30}$ ou que chaque paragraphe d'un certain discours débute par «Désormais... "»

Il faut dire que le «Désormais..." s'insère de différentes manières au sein de l'intrigue. Les auteurs l'emploient selon trois modes que nous appelons la citation, l'essentialisation et l'appropriation. Dans le premier cas, l'auteur rapporte les propos de Paul Sauvé, utilisant pour cela les guillemets ou le tiret: «Il n’hésite pas à amorcer l’opération déblocage sur

23. G.-É. Lapalme, Mémoires..., op. cit., 277; Denise Bombardier, Les «cent jours» du gouvernement Sauvé, mémoire de maîtrise (science politique), Université de Montréal, 1971, 16.

24. D. C. Thomson, Jean Lesage..., op. cit., 78; R. Prévost, «Le "désormais"... », loc. cit., 26.

25. Rae Murphy, Robert Chodos et Nick Auf Der Maur, Brian Mulroney, the boy from Baie-Comeau (Toronto, Lorimer, 1984), 43 ; Jill R. Mac Dougall, Performing Identities on the Stages of Quebec (New York, Peter Lang, 1997), 33.

26. Pierre Godin, Daniel Johnson. Tome 1: 1946-1964: La passion du pouvoir (Montréal, Éditions de l’Homme, 1980), 126; P. Labonne, Paul Sauvé..., op. cit., 87.

27. Jacqueline Cardinal et Laurent Lapierre, Pierre Jeanniot - Aux commandes du ciel (Montréal, Presses de l’Université du Québec, 2009), 188.

28. Louis-Philippe Audet, Histoire de l'enseignement au Québec. Tome 4: 1840-1971 (Montréal, Holt, Rinehart et Winston, 1971), 387; Jean-Pierre Charland, À l'aube du XXI siècle. Histoire du Québec et du Canada (Montréal, Lidec, 1997), 468.

29. Chantal Bouchard, La langue et le nombril: histoire d'une obsession québécoise (Saint-Laurent, Fides, 1998), 217; Philippe Séguin, Plus français que moi, tu meurs! France, Québec: des idées fausses à l'espérance partagée (Paris et Montréal, Albin Michel et VLB éditeur, 2000), 117.

30. Gérard Pelletier, Souvenirs. Tome 2 : Le temps des choix, 1960-1968 (Montréal, Stanké, 1986), 34-35 ; Laurent Laplante, «Kim Campbell : à quand son “désormais” ?”, Le Droit, 27 juillet 1993, 13.

31. Claude Bélanger, "Paul Sauvé», Quebec History, faculty.marianopolis.edu/c.belanger/quebechistory/ [consulté le 8 septembre 2013]; Bachir Mazouz et Benoît Tremblay, «Toward a Postbureaucratic Model of Governance: How Institutional Commitment Is Challenging Quebec's Administration », Public Administration Review, 66 (mars-avril 2006): 265 et 271. 
le plan administratif et à manifester son désir de rompre avec la politique de son prédécesseur en utilisant une formule qui a fait fureur à l'époque : "Désormais..."32.»; «Dès le premier caucus du parti, Sauvé annonce la couleur:- Désormais, je veux que chacun des ministres prennent [sic] ses responsabilités ${ }^{33}$.» Dans le second cas, le «Désormais...» devient un phénomène en soi, tel un concept, une idée dont font état les auteurs: «L'époque nouvelle commençait avec lui. Pendant la période dite des "Cent jours" de cet automne 1959, il allait pratiquer la politique du "Désormais...", les choses allaient se passer ainsi ${ }^{34}$ !» Enfin, dans le troisième cas, les auteurs s'approprient le terme pour paraphraser Paul Sauvé ou son gouvernement: «Désormais les subventions seront distribuées, partout où c'est possible, selon des normes invariables. Désormais, les universités auront des budgets stables ${ }^{35}$.»

Une autre différence notable consiste dans l'écriture même du "Désormais...». On le retrouve sous diverses formes dans la littérature: "désormais ${ }^{36}$ », «Désormais ${ }^{37}$ », «Désormais... ${ }^{38}$ », désormais ${ }^{39}$, Désormais ${ }^{40}$, Désormais... ${ }^{41}$, sans compter les italiques ${ }^{42}$. Pour notre part, nous nous sommes arrêté à une certaine forme, car elle nous apparaît comme la plus représentative du mythistoire. L'adverbe est ainsi entre guillemets, afin de bien indiquer qu'il s'agit d’une parole de Paul Sauvé. La lettre «d» est en majuscule, du fait qu'il aurait débuté ses discours par cet adverbe selon la version la plus courue de la légende. Enfin, les points de suspension soulignent à la fois l'absence et l'inutilité du reste de son discours. Du moment que le «Désormais...» a été prononcé, tout a été dit, en somme.

Quelques variables peuvent ainsi être introduites, certains éléments manquer à l'appel, mais la structure reste la même. Les trois citations placées en exergue de cet article le montrent bien. Toutes trois suivent en effet le même patron narratif. Sans nécessairement parler de la Grande

32. J. Hamelin et A. Garon, "Introduction... », loc. cit., 12.

33. P. Godin, Daniel Johnson..., op. cit., 126.

34. G. Bergeron, Pratique de l'État..., op. cit., 68.

35. André Laurendeau, Ces choses qui nous arrivent: chronique des années 1961-1966 (Montréal, HMH, 1970), 2.

36. D. Bombardier, Les «cent jours»..., op. cit., 16-18.

37. Conrad Black, Duplessis. Tome 2: Le pouvoir (Montréal, Éditions de l’Homme, 1977), 598.

38. J. Hamelin et A. Garon, "Introduction... », loc. cit., 12.

39. Dominique Clift, Le déclin du nationalisme au Québec (Montréal, Libre Expression, 1981), 28.

40. Réjean Tremblay, «Les 100 ans du Canadien - Désormais», La Presse, 5 décembre 2009, S3.

41. A. Laurendeau, Ces choses qui nous arrivent..., op. cit., 2.

42. Robert Prévost, «Désormais... - Paul Sauvé», Petit dictionnaire des citations québécoises (Montréal, Libre expression, 1988), 104. 
Noirceur duplessiste, de la Révolution tranquille ou du décès de Maurice Duplessis, le «Désormais...» de Paul Sauvé symbolise pour l'un comme pour l'autre une rupture entre deux temps et deux mondes.

Cette rupture qu’incarne le «Désormais...», nul ne l’a mieux cernée et ne s'y est intéressé sur une aussi longue période que Jocelyn Létourneau. Il importe de s'y arrêter, afin de dégager trois moments distincts de son parcours. Dans une série d'articles publiés entre 1989 et 1992, il s'est intéressé au récit de la technocratie qui a fondé l'histoire du Québec moderne sur sa mémoire. Ce récit, soutient Létourneau, s’appuie sur la dichotomie Grande Noirceur/Révolution tranquille où le «Désormais...» est appelé à remplir un rôle clé. Renvoyant aux travaux de Dorval Brunelle, Létourneau note: «On sait à quel point le vocable "désormais", prononcé par Paul Sauvé (successeur intérimaire de Maurice Duplessis à la tête du Québec), est utilisé par les analystes pour qualifier toute cette période de "proto-changement" que constituent les cent jours de son règne ${ }^{43}$." Létourneau se fait ici très critique de cette interprétation des analystes qui vient renforcer la dichotomie.

Dans un autre article, l'historien de l'Université Laval consacre au «Désormais...» de belles lignes, dans le style relevé qu’on lui connaît, afin de dégager les significations qui lui sont attachées:

Le vocable «désormais», célèbre dans la glose technocratique, fut prononcé par Paul Sauvé, Premier ministre du Québec à la suite du décès de Maurice Duplessis, survenu en septembre 1959. [...] Disons que cette fameuse expression est porteuse de significations particulières. Nous l'interprétons comme une magistrale excuse professée par la collectivité québécoise à l'égard d'elle-même. Elle est reniement de ce que cette collectivité était précédemment. Elle est exorcisation d'une époque et de sa figure centrale, Duplessis. Elle est prétexte à l'oubli d'un état antérieur malheureux. Elle est délivrance fondatrice d'un ordre nouveau. Elle est cri de ralliement d'une communauté communicationnelle. Elle marque l'avènement d'une nouvelle temporalité bienheureuse: le temps de la malchance et de l'empêchement est terminé, le poids du passé est tombé ${ }^{44}$.

Son regard n'est pourtant plus le même, alors qu'il semble louvoyer entre la posture de l'historien du discours et celle de l'essayiste, entre

43. Jocelyn Létourneau, «L'histoire du Québec d’après-guerre et mémoire collective de la technocratie », dans Diane Vincent, dir., Des analyses de discours (Québec, CÉLAT, 1989), 20; Jocelyn Létourneau, "Québec d'après-guerre et mémoire collective de la technocratie », Cahiers internationaux de sociologie, 90 (janvier-juin 1991): 79.

44. Jocelyn Létourneau, "Le "Québec moderne” : un chapitre du grand récit collectif des Québécois», Revue française de science politique, 42,5 (octobre 1992): 775 ; J. Létourneau, "La saga... », loc. cit., 63. 
l'analyse critique et l'interprétation. Est-ce ce que Létourneau observe au fil de ses analyses, dont il nous livre ici en quelque sorte la synthèse, dans ses propres mots? Ou n'est-ce pas plutôt ainsi que Létourneau interprète lui-même le "Désormais... », s'inscrivant à ce titre dans la lignée des analystes qui renforcent le récit technocratique? À ce stade-ci, il est difficile de le dire.

Dans ses écrits plus récents, Létourneau a eu l'occasion de revenir sur le «Désormais...», d'abord dans sa synthèse historique : "C'est cette transition douce mais convaincue, inaugurée par un retentissant "Désormais", qu'amorce Paul Sauvé, successeur désigné à Duplessis, durant un mandat écourté à cent jours par sa mort subite ${ }^{45}$.» En 2010, il est appelé à produire un texte sur le cinquantième anniversaire de la Révolution tranquille pour le compte du magazine The Walrus, dont nous reproduisons ici la traduction française :

Le décès de Duplessis, en septembre 1959, modifie le rapport de force en faveur du changement. «Désormais», dit Paul Sauvé, successeur du "Chef » à la tête de l'Union nationale, la modernisation du Québec sera prioritaire. Fauché par la mort en pleine ascension, Sauvé ne mènera sa petite révolution que durant quatre mois. C'est pourtant avec l'arrivée de cet homme au pouvoir $[\ldots]$ que le bousculement s'amorce, qui deviendra basculement ${ }^{46}$.

Le revirement de situation est complet: Létourneau s'est approprié la posture qu'il critiquait jadis chez ses confrères. Ce qu'il observait dans la production historienne québécoise, vingt ans auparavant, il le reproduit dans ses propres textes des années 2000. Alors qu'il reprend la glose technocratique, il applique la même grille de lecture, faisant du «Désormais... » l'icône du "proto-changement " pour reprendre son concept, insistant sur le fait que Paul Sauvé a bel et bien dit «Désormais...», ce sur quoi il ne se prononçait pas dans ses premiers textes. Le cheminement de Létourneau sur la question est d'autant plus intéressant que Sauvé n’a jamais prononcé le «Désormais...». Il est temps de revenir sur cette question, essentielle à notre propos, et de revenir sur les éléments qui nous permettent d'aller de l'avant avec cette affirmation.

45. Jocelyn Létourneau, Le Québec, les Québécois : un parcours historique (Saint-Laurent, Fides, 2004), 78.

46. Jocelyn Létourneau, «La Révolution tranquille comme lieu de mémoire», The Walrus, octobre 2010, thewalrus.ca [consulté le 5 septembre 2013]. 


\section{«DÉSORMAIS... », CE MOT QUE PAUL SAUVÉ N'A JAMAIS PRONONCÉ}

Il importe de souligner que d'autres avant nous ont émis des réserves à l'endroit du "Désormais... ». Dès 1971, Denise Bombardier souligne «que Paul Sauvé, contrairement à ce que l'on croit, n'a jamais débuté un discours avec l'expression "désormais", il n’a jamais insisté sur ce terme de manière à en faire sa "marque de commerce". Ce sont les journalistes qui lui ont collé l'étiquette ${ }^{47}$.» Une remarque que d'autres reprendront à sa suite $^{48}$. Or, elle indique que Sauvé «se contenta d’insérer dans quelquesunes de ses déclarations ses fameux "désormais" qui indiquent une certaine rupture par rapport au passé encore récent ${ }^{49}$ ", pour ensuite affirmer, dans un autre texte, qu'il «aimait» dire "Désormais... »". Enfin, dans un documentaire paru en 2008, Paul Carvalho et Paul Labonne affirment, sans toutefois le démontrer, qu'aucun "discours de Paul Sauvé, écrit ou enregistré, ne mentionne le mot "désormais". Pourtant, l'expression si souvent entendue par [Fernand] Dostie et [Jean] Pelletier, résume l'esprit d'une époque ${ }^{51}$.»

Fernand Dostie était le chef de cabinet de Paul Sauvé et Jean Pelletier, son attaché de presse. D’autres contemporains mentionnent le "Désormais... " : c'est le cas de François-Albert Angers ${ }^{52}$, Lionel Bertrand ${ }^{53}$, Roch Bolduc $^{54}$, Claude Castonguay ${ }^{55}$, Jean Cournoyer ${ }^{56}$, Claude Jean Devirieux ${ }^{57}$, Léon Dion ${ }^{58}$, Henri Dutil ${ }^{59}$, André Forcier ${ }^{60}$, Gérard Filion ${ }^{61}$, Jean-Louis

47. D. Bombardier, Les «cent jours»..., op. cit., 16 .

48. Pierre Duchesne, Jacques Parizeau. Tome 1: Le Croisé 1930-1970 (Montréal, Québec/Amérique, 2001), 211 ; Jacques Lacoursière, Histoire populaire du Québec. Tome 5 : 1960-1970 (Québec, Septentrion, 2008), 33-35.

49. D. Bombardier, Les «cent jours»..., op. cit., 164.

50. Denise Bombardier, «Les cent jours de Paul Sauvé», Magazine MacLean, 15,1 (janvier 1975): 19.

51. P. Carvalho et P. Labonne, Paul Sauvé..., op. cit.

52. François-Albert Angers, "Les relations fédérales-provinciales sous le régime Duplessis», dans Alain-G. Gagnon et Michel Sarra-Bournet, dir., Duplessis : entre la Grande Noirceur et la société libérale (Montréal, Québec/Amérique, 1997), 240.

53. L. Bertrand, Quarante ans..., op. cit., 79.

54. R. Bolduc, Le Mandarin..., op. cit., 101.

55. C. Castonguay, Mémoires..., op. cit., 38.

56. Jean Cournoyer, participation à l'émission Bazzo.tv, épisode 260, 2010, www.zonevideo.telequebec.tv/ [consulté le 16 septembre 2013].

57. Claude Jean Devirieux, Derrière l'information officielle 1950-2000 (Québec, Septentrion, 2012), 231.

58. L. Dion, Québec 1945-2000..., op. cit., 16 et 142.

59. Henri Dutil, «Témoignage», dans Robert Comeau, dir., Jean Lesage et l'éveil d'une nation (Sillery, Presses de l’Université du Québec, 1989), 15.

60. Normand Provencher, «Entrevue - André Forcier - Souvenirs amers », Le Soleil, 28 février 2009, A25.

61. Mario Cardinal, Vincent Lemieux et Florian Sauvageau, Si l'Union nationale m'était contée... (Sillery, Boréal Express, 1978), 45. 
Gagnon $^{62}$, Paul Gérin-Lajoie ${ }^{63}$, Claude-Gilles Gosselin ${ }^{64}$, Lionel Groulx ${ }^{65}$, Georges-Émile Lapalme ${ }^{66}$, Pierre Laporte ${ }^{67}$, André Laurendeau ${ }^{68}$, Jean Lesage $^{69}$, René Lévesque ${ }^{70}$, Louis O’Neill ${ }^{71}$, Jacques Parizeau ${ }^{72}$, Gérard Pelletier $^{73}$, Robert Prévost ${ }^{74}$ et Arthur Tremblay ${ }^{75}$. Distinguons d'emblée les témoins des contemporains, c'est-à-dire ceux qui ont entendu Paul Sauvé prononcer le "Désormais...» de ceux qui le relatent. De cette même liste, nous ne conservons plus que Jean Cournoyer, Fernand Dostie, Claude-Gilles Gosselin, Pierre Laporte, Jean Pelletier et Robert Prévost.

Les témoignages de ces acteurs - hommes politiques, journalistes, artistes - sont assez semblables. De prime abord, ils sont pour la plupart avares de détails, ne mentionnant pas nécessairement l'endroit ou le moment. De fait, on reste dans le vague: leur souvenir se limite au seul mot. Ils se souviennent tous, sans exception, que Paul Sauvé a prononcé le «Désormais...». Mais ensuite? Plus rien. De son côté, Robert Prévost affirme que le «Désormais...» a été prononcé par Paul Sauvé lors de sa première conférence de presse, le 11 septembre. Il y était. Or, aucun journaliste ne rapporte cet adverbe dans les jours qui suivent. Pour sa part, l'ancien député Claude-Gilles Gosselin rapporte que son chef n'avait que ce mot à la bouche lors des caucus du parti. Fernand Dostie et Jean Pelletier abondent dans le même sens. Si c'était bel et bien le cas, si le "Désormais..." n'avait pas relevé du domaine public mais bien du domaine privé, que dire alors de Pierre Laporte et Jean Cournoyer qui, tous deux, spécifient avoir été là, le jour où Paul Sauvé a prononcé le «Désormais...»? Le premier rapporte la chose en 1962, à l'Assemblée législative, le second en 2010, à l'émission de Marie-France Bazzo à TéléQuébec. Comme on peut le voir, ces témoignages se contredisent, s’inva-

62. J.-L. Gagnon, Les Apostasies..., op. cit., 16.

63. P. Gérin-Lajoie, Combats..., op. cit., 173.

64. Entrevue avec Claude-Gilles Gosselin pour la Bibliothèque de l'Assemblée nationale, 2010. Inédit.

65. Lionel Groulx, Mes mémoires. Tome 4: 1940-1967 (Montréal, Fides, 1974), 336-337.

66. G.-É. Lapalme, Mémoires..., op. cit., 277-278.

67. Assemblée nationale du Québec, Débats de l'Assemblée législative du Québec, Troisième session, $26^{e}$ législature, mardi le 3 avril 1962.

68. Candide, «Désormais, dit-il», Le Devoir, 5 novembre 1959, 4.

69. M. Cardinal, V. Lemieux et F. Sauvageau, Si l'Union nationale..., op. cit., 45.

70. René Lévesque, Attendez que je me rappelle... (Montréal, Québec/Amérique, 1986), 199.

71. Louis O’Neill, Les trains qui passent: propos et souvenirs d'un citoyen libre (Montréal, Fides, 2003), 114.

72. Jacques Parizeau, Pour un Québec souverain (Montréal, VLB éditeur, 1997), 12-13.

73. G. Pelletier, Souvenirs..., op. cit., 34-36.

74. R. Prévost, "Le “désormais" ...", loc. cit., 21-28.

75. Robert Bothwell, Canada and Quebec: one country, two histories (Vancouver, UBC Press, 1995), 102-103. 
lident en quelque sorte l'un l'autre. Cela ne prouve toutefois pas que Paul Sauvé n’a jamais prononcé le «Désormais...»; il nous revient d'en faire la démonstration. Pour ce faire, il nous faut revenir sur nos pas.

Avant d'en arriver à la conclusion que Paul Sauvé n'avait jamais prononcé le «Désormais...», nous sommes d'abord parti à la recherche de ce terme. Pour ce faire, nous avons consulté les débats reconstitués de la $4^{\mathrm{e}}$ session de la $25^{\mathrm{e}}$ législature, des enregistrements audiovisuels de Paul Sauvé et de personnalités à l'occasion de son assermentation et de son décès, dans le Fonds Famille Sauvé à Bibliothèque et Archives nationales du Québec, et dépouillé des journaux de l'époque ${ }^{76}$. Notre démarche était assez simple. Nous étions à la recherche d'une déclaration qui ne laisse place à aucune équivoque. En d'autres termes, nous voulions soit entendre Sauvé dire ce mot, soit retrouver une déclaration de Sauvé citée entre guillemets, où le journaliste aurait alors reproduit le verbatim de ses propos. Non seulement n'avons-nous pu trouver la moindre citation où il aurait prononcé le vocable, mais bien au contraire, Sauvé cultive les déclarations où il rappelle son attachement indéfectible à Duplessis. Nous y reviendrons. Cela étant, se pourrait-il, comme le soutient Prévost, que Sauvé ait prononcé le «Désormais... » lors d'un discours ou d'une conférence de presse sans que cela soit rapporté tel quel par les journalistes? Disons que c'est on ne peut plus improbable.

Dès le lendemain de l'assermentation de Paul Sauvé, le 12 septembre 1959, Le Devoir titre à la une, sous la plume de Pierre Laporte: «Québec: l'atmosphère est complètement changée ${ }^{77}$ !» Et ce, en moins de 24 heures. Or, en parcourant l'article, on peut toutefois y lire que le «nouveau premier ministre ne veut pas rompre avec le passé». De fait, si le premier ministre avait laissé échapper un "Désormais... », un "dorénavant» ou quelque autre terme de ce genre, il ne fait pas de doute à notre esprit que les journalistes auraient rapporté ce terme. Sans compter que les journalistes ne sont pas les seuls à guetter les moindres faits et gestes, les moindres paroles du nouveau premier ministre. Les libéraux le font également. C’est ici qu'une étude minutieuse des débats reconstitués de l'Assemblée législative prend toute son importance.

Ce qu'il faut comprendre de cette session parlementaire, c'est que la stratégie de l'opposition libérale est fort simple: elle consiste à prendre

76. Il s'agit des journaux Le Devoir, La Presse, Le Soleil, Notre Temps, L'Événement-Journal, L'Action catholique, Montreal Star, Montreal Gazette, Toronto Star et The Globe and Mail.

77. Pierre Laporte, "Québec: l'atmosphère est complètement changée!», Le Devoir, 12 septembre $1959,1$. 
Sauvé en défaut vis-à-vis de son prédécesseur afin de le brouiller avec sa base électorale $^{78}$. Pour parvenir à leurs fins, les libéraux sont aux aguets, et en Chambre, ils tenteront de faire dire à Paul Sauvé non pas qu'il renie, mais bien qu'il se détache de Maurice Duplessis ${ }^{79}$. La session parlementaire s'ouvre sur un Georges-Émile Lapalme en forme, qui accuse le premier ministre de faire la déstalinisation du régime duplessiste. Mais Sauvé est plus fin encore que ses adversaires. Il réplique coup sur coup, se joue par moments d'eux et parvient à tirer son épingle du jeu, notamment lorsqu'il permet le débat sur le scandale de la Corporation du gaz naturel, le 26 novembre, alors que l'affaire est $s u b$ judice ${ }^{80}$. Dans ce contexte, on comprend pourquoi Sauvé ne prononce pas en Chambre un marqueur de rupture temporelle tel que "désormais»: les libéraux s'en seraient emparés sur-le-champ pour s'en servir contre lui.

En fait, dans ses discours prononcés en Chambre, tels que rapportés dans les débats reconstitués, Paul Sauvé aurait échappé ce vocable à une seule occasion en tant que premier ministre. Le 14 décembre 1959, en comité plénier sur l'étude du projet de loi de l'autoroute MontréalLaurentides, le député libéral de Westmount-Saint-Georges, John Richard Hyde, suggère de changer la loi en ce qui concerne la nomination du personnel. À cette occasion, Paul Sauvé use certes dudit vocable, mais devant l'attitude caustique des députés de l'opposition, qui le narguent à propos de ses rapports avec son prédécesseur, le premier ministre se rebiffe et réplique, cinglant. Il ne permet pas qu'on l'attaque sur ce flanc, quitte à rebrousser chemin et à s'enfermer dans ses positions. Nous reproduisons l'échange dans son intégralité:

M. Hyde (Westmount-Saint-Georges) émet une objection à propos de la nomination des membres du personnel de l'autoroute. La loi dit que c'est le lieutenant-gouverneur en conseil qui nomme le personnel.

L'honorable M. Talbot (Chicoutimi): C'est l'Office qui recommande les nominations.

M. Hyde (Westmount-Saint-Georges) suggère de changer la loi.

78. Voir Alexandre Turgeon, «Introduction historique $-4^{\mathrm{e}}$ session de la $25^{\mathrm{e}}$ Législature (du 18 novembre 1959 au 18 mars 1960)", Débats reconstitués de l’Assemblée législative, Assemblée nationale, Québec, 2010, p. vii-xlviii.

79. «M. Lesage: L’U.N. a renié Duplessis mais elle conserve le duplessisme», Le Devoir, 19 octobre 1959, 3.

80. Voir Alexandre Turgeon, «Pour s'assurer une place dans l’histoire: Paul Sauvé et la lutte à la corruption (1959)", Histoire Engagée (février 2012), www.histoireengagee.ca [consulté le 17 septembre 2013]. 
L'honorable M. Sauvé (Deux-Montagnes) Quand la loi a été faite, on s'est préoccupé surtout du côté administratif de l'affaire. Cette proposition semble raisonnable.

Il (l'honorable M. Sauvé) propose de rapporter le projet et dit qu'il préparera un amendement pourvoyant à ce que ce soit l'Office qui nomme désormais les employés et non le lieutenant-gouverneur en conseil.

M. Hyde (Westmount-Saint-Georges) : L'an dernier, lors de l'étude de la loi de l'autoroute, l'opposition a proposé un amendement dans le même sens que celui que veut apporter maintenant le premier ministre.

L'honorable M. Sauvé (Deux-Montagnes) demande aux membres de l'opposition s'ils veulent qu'il s'excuse de son attitude antérieure.

M. Hamel (Saint-Maurice): L'ancien premier ministre (M. Duplessis) avait alors déclaré que l'amendement de l'opposition n'avait pas de bon sens.

L'honorable M. Sauvé (Deux-Montagnes): J'ai assez de respect pour sa mémoire pour penser maintenant que, s'il a dit ça, c'est qu'il avait raison. Aucun amendement ne sera apporté. On laisse tout cela comme c'est. Êtes-vous contents ${ }^{81}$ ?

Un autre cas intéressant concerne un discours prononcé par Sauvé à l'Université de Montréal. Le «Désormais... y y serait associé aux politiques du gouvernement québécois en matière d'éducation. Dès 1966, Michel Brunet rapporte que c'est «à l'Université de Montréal, au mois d'octobre 1959, que Paul Sauvé annonça que “désormais" l'État provincial s’acquitterait de toutes ses responsabilités dans l'organisation et le financement de l'enseignement supérieur ${ }^{82}{ }^{\prime}$. Trois ans plus tard, Louis-Philippe Audet tient des propos similaires, à la différence qu'il situe ce discours en septembre 1959: “"Désormais", dira le nouveau Premier Ministre, ici même, à l'Université de Montréal, invitant ainsi la Province à se tourner plutôt vers l'avenir et à oublier le passé. C'est à ce moment, nous semble-t-il, qu’il faut placer le début de la réforme scolaire ${ }^{83}$.» En 1997, Jean-Pierre Charland souligne que Paul Sauvé «se démarque du "chef" en commençant un discours prononcé à l'Université de Montréal par le mot "Désormais" ${ }^{84}$ ", tandis que Jacques Lacoursière, sans toutefois préciser le

81. Assemblée nationale du Québec, Débats de l'Assemblée législative du Québec, Quatrième session, $25^{\mathrm{e}}$ législature, lundi le 14 décembre 1959, 270. Nous soulignons.

82. Michel Brunet, «L'enseignement supérieur: les difficultés et les défis », L'inter. Mensuel d’information des Diplômés de l'Université de Montréal (avril-mai 1966): 5.

83. Louis-Philippe Audet, Bilan de la réforme scolaire au Québec, 1959-1969, Université de Montréal, 12 février 1969, 13; voir aussi L.-P. Audet, Histoire de l'enseignement..., op. cit., 387.

84. J.-P. Charland, À l'aube..., op. cit., 468. 
lieu, affirme que «Sauvé annonce, le 26 octobre, qu'à l'avenir "les demandes des universités seront examinées de façon objective par le Conseil des ministres". (La Presse, même jour) ${ }^{85}$ ". Qu'en est-il? Qu'a dit Paul Sauvé au juste, et quand l'aurait-il dit?

C'est le 24 octobre 1959 que Paul Sauvé prononce un discours à l'Université de Montréal pour annoncer la nouvelle politique du gouvernement québécois en ce qui a trait au financement des universités. Deux articles reproduisent de larges extraits du discours de Sauvé à la une le 26 octobre, l'un dans Le Devoir, l'autre dans La Presse. Selon Michel Roy du Devoir, le premier ministre aurait déclaré : «À l'avenir, les demandes des universités seront examinées de façon objective par le Conseil des ministres ${ }^{86}$.» Pour sa part, Renaude Lapointe de La Presse rapporte plutôt la chose de cette manière: "Dans l'avenir, les demandes faites par votre université et les autres seront examinées par le Conseil exécutif, sans préjugés, en vue de servir les fins énumérées par vous ${ }^{87}$.»

Se pourrait-il que le "Désormais... » soit en fait une méprise, et que ce soit "À l'avenir» que Paul Sauvé eut prononcé? Trois choses. Premièrement, il faut noter la subtile différence entre les deux comptes rendus. «À l'avenir » et «Dans l'avenir» ne sont pas des synonymes. Selon l'Office québécois de la langue française, le premier est «synonyme de désormais, de dorénavant [et] se dit en parlant d'un changement et suggère généralement que les choses n'ont pas été faites correctement jusqu'à maintenant", tandis que le second "renvoie à un moment à venir et est synonyme de un jour prochain, ultérieurement ${ }^{88}$ ". Deuxièmement, des deux comptes rendus du discours de Paul Sauvé, c'est celui de Renaude Lapointe qui est le plus complet, celui qui nous apparaît comme le plus fiable. Le lecteur sent moins la présence du narrateur qui s'immisce à l'occasion dans les propos de Sauvé. Qu'à cela ne tienne: il se pourrait que Roy ait vu juste, sur ce point, et que le premier ministre ait bel et bien dit «À l'avenir». Or, en troisième lieu, il faut considérer la nature du "Désormais...». Il n'est pas unique, et ce ne peut être une erreur de parcours, loin de là. Que ce soit dans les récits des témoins ou dans les travaux des chercheurs, une

85. J. Lacoursière, Histoire populaire..., op. cit., 37.

86. Michel Roy, «M. Paul Sauvé annonce une politique nouvelle de financement pour les universités du Québec», Le Devoir, 26 octobre 1959, 6. Un extrait que "note avec amusement» l'avocat Jean-L. Péloquin, «Paul Sauvé, vieux régime, nouvelle face», Le Devoir, 7 novembre 1959, 4.

87. Renaude Lapointe, «L'hon. Paul Sauvé annonce sa formule - Le financement des universités du Québec est désormais assuré », La Presse, 26 octobre 1959, 28.

88. "Futur et avenir», Office québécois de la langue française, www.oqlf.gouv.qc.ca/ [consulté le 18 septembre 2013]. Les italiques sont dans l'original. 
constante demeure: il s'agit non pas de l'exception qui confirme la règle - comme semble être le cas de "À l'avenir»-, mais bien de la règle ellemême. C'est ce qui nous fait dire, en bout de ligne, que Paul Sauvé n'a jamais prononcé le «Désormais...».

Comme nous l'avons déjà avancé, le «Désormais...» de Paul Sauvé est un mythistoire, c'est-à-dire que le fictif passe pour vrai. Mais ce fictif, d'où vient-il? Qui est son auteur? Est-il individuel ou collectif ? Pour répondre à ces questions, notre regard doit maintenant se tourner vers les journaux, comme d'autres l'ont indiqué, mais plus spécifiquement encore vers Le Devoir.

\section{ANDRÉ LAURENDEAU ET LA FORMATION DU MYTHISTOIRE}

Tout commence avec les journalistes qui couvrent les activités du gouvernement Sauvé, ceux du Devoir en particulier, et qui vont user du vocable pour rendre compte de ses faits et gestes où, parfois, les comptes rendus constituent un amalgame de déclarations du premier ministre insérées dans leur narration. Dès le 12 septembre 1959, soit le lendemain de l'assermentation de Paul Sauvé, deux articles ont recours à l'adverbe pour annoncer qu'Antoine Rivard, «ministre des transports, serait désormais chargé du département du procureur général ${ }^{89}$ » et que "[d]ésormais les journalistes de la radio et de la télévision seront traités sur le même pied que ceux de la presse ${ }^{90} \%$.

Les hommes politiques ne sont pas en reste. Dans un discours prononcé devant la Fédération libérale du Québec et reproduit dans les pages du Devoir, Jean Lesage s'en prend durement à Maurice Duplessis, mais Paul Sauvé demeure sa véritable cible: "Qui donc reconnaît maintenant que M. Duplessis brimait nos libertés? Nul autre que le nouveau chef du vieux régime. Dès le lendemain des obsèques de $\mathrm{M}$. Duplessis, M. Sauvé déclarait que dorénavant les journalistes auraient accès à toutes les informations de l'administration provinciale afin de pouvoir renseigner le public $^{91}$ !» Bien qu'il évoque une déclaration de Sauvé, qu'on ne s’y trompe pas: c'est Lesage qui parle, qui insiste sur le mot "dorénavant»; d'où le fait que ce mot est en caractère gras dans l'article.

Pour sa part, Renaude Lapointe emploie le terme «désormais» dans le titre de son article de La Presse, alors qu'elle revient sur les annonces du

89. «En moins de 24 heures, M. Sauvé passe à l'action», Le Devoir, 12 septembre 1959, 1.

90. «Relations cordiales entre M. Paul Sauvé et les journalistes», Le Devoir, 12 septembre 1959, 2.

91. "M. Lesage: L’U. N. a renié Duplessis mais elle conserve le duplessisme», Le Devoir, 19 octobre 1959, 3. Caractères gras dans l'original. 
gouvernement en matière d'éducation, dans le cas que nous venons de présenter ${ }^{92}$. Le 29 octobre, trois textes du Devoir ont recours au «désormais». On peut ainsi lire à la une que «M. Sauvé a d'autre part annoncé que tous les sous-ministres seront désormais payés $\$ 14.000$ par année $e^{93}$ », que les «travailleurs spécialisés, les ingénieurs, les architectes, les techniciens, a-t-il précisé, recevront désormais des salaires comparables aux échelles de l’État fédéral ou de l'industrie» et qu'enfin, «[D]orénavant, a-t-il dit, les fonctionnaires seront payés suivant leur valeur et leur compétence ${ }^{94} »$. André Laurendeau ferme la marche dans un éditorial, alors qu'il revient sur le discours de Sauvé prononcé à l'Université de Montréal: «Désormais, [les universités] savent à temps combien elles recevront de l'État provincial, et quand l'argent leur parviendra; elles savent surtout que cette subvention, dont le caractère est continu, sera suffisante. » Selon Laurendeau, l'une «des conséquences les plus heureuses de ce geste, c'est que désormais l'on pourra aborder plus directement les problèmes universitaires ${ }^{95}$ ». Cet usage répété du terme par l'éditorialiste n'est pas anodin.

Une semaine plus tard, soit le 5 novembre 1959, Laurendeau signe sous son pseudonyme Candide un encadré intitulé «Désormais, dit-1 ${ }^{196}$ ». Ce texte est important car il s'agit de la première occasion où le «Désormais...» est attribué directement et sans ambages à Paul Sauvé. On retrouve quinze «Désormais...» dans ce texte, Dès le départ, et c'est en soi assez remarquable, Laurendeau définit le «Désormais...» tel qu’on le retrouvera par la suite dans la littérature:

Le mot éclate sans préparation. Il n'est précédé ni d'un mais ni d'un cependant avertisseur. Il se tient solidement sur ses trois pattes: dé-sor-mais. Il a comme une valeur propre. En lui se résorbent d'apparentes contradictions. C'est un jugement implicite et discret, et c'est en même temps une promesse formelle.» L'éditorialiste l'utilise également dans ce texte afin de subsumer sous ce terme quelques-unes des plus importantes politiques de Sauvé: «Désormais, les fonc-

92. R. Lapointe, «L'hon. Paul Sauvé... », loc. cit., 1 et 28.

93. "M. Dostie, chef de cabinet», Le Devoir, 29 octobre 1959, 1.

94. «Le premier ministre signe l'arrêt ministériel - \$7 millions aux fonctionnaires ", Le Devoir, 29 octobre 1959, 1. Cet extrait semble indiquer que Sauvé aurait ici employé le terme «dorénavant», synonyme de «désormais». Or, en consultant l'article, on constate que ce n'est pas le cas. Prenons le paragraphe d'où est tirée cette citation. Seul un court extrait est entre guillemets, ce qui indique que cet extrait en particulier est le verbatim des propos de Sauvé: «Le chef du gouvernement a reconnu que les fonctionnaires étaient mal payés depuis plusieurs années. Dorénavant, a-t-il dit, les fonctionnaires seront payés suivant leur valeur et leur compétence. Il aura fallu, a-t-il ajouté, que le personnel de la Commission du service civil travaille "presque jour et nuit" pour accomplir cette tâche de révision en trois semaines. »

95. André Laurendeau, "Quand l’université pourra respirer», Le Devoir, 29 octobre 1959, 4.

96. Candide, «Désormais...», loc. cit., 4. 
tionnaires seront mieux payés. Désormais, les subventions aux universités seront statutaires. Désormais la législation municipale sera simplifiée. Désormais ${ }^{97} \ldots$

Le 13 novembre, Laurendeau continue de plus bel, en revenant sur les différences entre Duplessis et son successeur: «M. Sauvé a un tempérament très différent. Il s'est montré jusqu'ici sage et habile. En deux mois, il a complètement modifié le paysage. Ses désormais, ses à l'avenir tombent comme des coups de hache sur ce qui reste de vieux embêtements duplessistes ${ }^{98}$.» Le «Désormais... » n’est pas seul dans ce texte. Il est ici accompagné d'un complice, "à l'avenir ${ }^{99}$ ", mais celui-ci ne passera pas à la postérité.

Le lendemain, un étudiant - c'est ainsi qu'il se présente, sans s'identifier davantage - fait état à son tour du "Désormais...». Répondant au texte de Candide, il lui indique avoir "trouvé un autre "désormais" qui [1]e laisse pensif ». Celui-ci aurait été prononcé par Roger Mathieu, président de la Confédération des travailleurs catholiques du Canada, alors qu'il rapportait «des paroles de M. Sauvé: "À l'avenir (donc désormais), la justice et l'équité auront la même signification pour tout le monde." Qu'est-ce à dire $^{100}$ ?" Qu'est-ce à dire, en effet? Cet étudiant aurait-il débusqué un autre "Désormais...", sous la forme d'un autre "À l'avenir» qui nous aurait échappé? Ce n'est pas tout à fait le cas. Cet étudiant fait référence à un texte du Devoir où Roger Mathieu revient sur sa rencontre avec Paul Sauvé: «Nous avons tous beaucoup apprécié l'assurance donnée par le premier ministre qu'à l'avenir la justice et l'équité auront la même signification pour tous ${ }^{101}$.»C'est Roger Mathieu qui interprète de cette manière, en ayant recours à l'expression "à l'avenir", les propos, voire les intentions de Paul Sauvé. Des propos que cet étudiant attribue, à tort, au premier ministre.

Un mois ne s'est pas écoulé que déjà des artisans du Devoir reviennent sur les implications, sur les effets du «Désormais... ». Selon Pierre Laporte, il s'agit d'"un mot en passe de devenir fameux», n'hésitant pas à l'employer lui-même dans le titre de son article ${ }^{102}$, alors qu'André Laurendeau

97. Id. Les italiques sont dans l'original.

98. André Laurendeau, «M. Sauvé déconcerte amis et adversaires», Le Devoir, 13 novembre 1959, 4. Les italiques sont dans l'original.

99. De toute évidence, André Laurendeau fait référence ici à M. Roy, «M. Paul Sauvé... », loc. cit., 1 et 6 .

100. Un étudiant, «Désormais», Le Devoir, 14 novembre 1959, 4.

101. "Le mémoire de la CTCC à Québec - Roger Mathieu: "Collaboration, oui! mais abandon des principes, jamais!"”, Le Devoir, 5 novembre 1959, 3.

102. Pierre Laporte, «"Désormais" l'Assemblée législative aura les documents prévus par les règlements", Le Devoir, 26 novembre 1959, 1. 
s’inquiète déjà, pour sa part, que Paul Sauvé "ne dit plus "désormais...”" . Il conclut d'ailleurs son éditorial sur ces mots : «M. Sauvé, cette fois, n’a pas dit désormais. Là-dessus, le duplessisme continue ${ }^{103}$.»

Deux autres articles du Devoir font état du «Désormais... », et ce ne sont pas les moindres. Ils nous permettent en fait de mieux comprendre de quoi il s'agit. Lors d'une séance au comité des bills publics, le journaliste écrit que «M. Sauvé consulte ses collègues!» - on notera le point d'exclamation qui signale l'inédit de la chose -, ce qui tranche radicalement avec la façon de faire de Duplessis. Le quotidien rapporte la chose sous le titre: "Encore un heureux "désormais"104...». Le 19 décembre, devant la décision de Paul Sauvé de revoir le bill 34 qui met un terme au système de l'énumérateur unique, dénoncé depuis longtemps par Georges-Émile Lapalme et bien d'autres, Le Devoir annonce: "Un "désormais" de taille - Le bill 34 : la clause relative à l'énumérateur unique est rayée ${ }^{105}$ !»

Ces deux articles illustrent de manière éloquente comment certaines décisions, comment certains actes de Sauvé ont pu être perçus comme marquant une rupture nette avec Duplessis. À ces actes, d'aucuns ont apposé le vocable "Désormais... " pour les immortaliser, sans que Sauvé n'ait toutefois prononcé le terme à ces occasions. Du vivant de Paul Sauvé, il s'agit d'ailleurs des seules sources à faire état du «Désormais... ». Toutes les autres pièces, écrites ou orales, qui le rapportent sont postérieures à son décès, survenu le 2 janvier 1960.

Au terme de cet exercice, une question toutefois demeure: pourquoi André Laurendeau fait-il dire «Désormais... » à Paul Sauvé? Pour répondre à cette question, il importe de rappeler dans quel contexte Paul Sauvé devient premier ministre, le 11 septembre 1959. À son arrivée au pouvoir, Sauvé doit composer avec un héritage controversé que lui a légué son prédécesseur, un héritage entaché lourdement par maints scandales. Il doit aussi ménager la base électorale de l'Union nationale, fidèle à Duplessis. Tout cela en affrontant une opposition libérale farouche, ragaillardie, qui sent que son heure est arrivée et qui est prête à en découdre avec ce gouvernement fatigué, usé par le pouvoir. Dans ce contexte pour le moins défavorable, Paul Sauvé s’est imposé en se révélant

103. André Laurendeau, "Où les “désormais” s’arrêtent», Le Devoir, 28 novembre 1959, 4. Les italiques sont dans l'original.

104. «Encore un heureux "désormais”... - M. Sauvé consulte ses collègues!», Le Devoir, 2 décembre 1959, 1-2.

105. "Un “désormais" de taille - Le bill 34 : la clause relative à l'énumérateur unique est rayée!», Le Devoir, 19 décembre 1959, 1. 
être un personnage apparemment contradictoire. D’un côté, par ses actes, Sauvé rompt avec Duplessis: les relations avec les journalistes sont normalisées, le bill 34 est aboli, les journaux rapportent qu'il est arrivé à une entente avec le gouvernement fédéral sur la question des universités, pour ne donner que ces exemples. Mais d'un autre côté, par ses paroles, Sauvé ne cesse de rappeler son attachement indéfectible à son prédécesseur. Il aime bien dire que la «voix de la province peut avoir changé et son ton peut avoir changé, mais [que] la province de Québec n’a pas changé», c'est-à-dire qu'il poursuit «simplement le travail entrepris» par Duplessis ${ }^{106}$. Il fait la démonstration, en fait, qu'il est une bête politique.

C'est un véritable tour de force que Paul Sauvé accomplit, et ce, dans de brefs délais. Quelques heures à peine après son assermentation, il parvient à incarner le changement. Ses manières de faire, sa présence et son ouverture subjuguent les journalistes présents, en particulier ceux du Devoir, pourtant très défavorables à sa cause a priori. Ce qui est remarquable, c'est que Sauvé y parvient non seulement sans renier, mais sans même s'éloigner, sans même se détacher un tant soit peu de Duplessis. Les libéraux vont chercher justement à le prendre en défaut sur cette question. Comme quoi, ce ne peut être que de deux choses l'une: soit Sauvé incarne le changement, c'est donc dire qu'il désavoue Duplessis; soit Sauvé ne désavoue pas Duplessis, c'est donc dire qu'il n'incarne en rien le changement. C'est là le tour de force de Paul Sauvé. Alors qu'il coupe l'herbe sous le pied des libéraux en appliquant pratiquement leur programme, le nouveau premier ministre répète à qui veut bien l'entendre qu'il ne fait que continuer l'œuvre de Maurice Duplessis, rassurant par le fait même sa base électorale, plus que satisfaite.

Paul Sauvé est parvenu à accomplir ce tour de force, il est vrai, mais au prix d'une contradiction qui semble indissoluble entre ses actes et ses discours. Par ses actes, il fait preuve de rupture, tandis que dans ses discours, il se fait l'avocat de la continuité. La contradiction pose problème aux observateurs de l'époque, et en particulier à André Laurendeau, qui ne savent plus sur quel pied danser lorsqu'ils sont appelés à expliquer, à rationaliser son comportement. C'est là qu'intervient le «Désormais...».

106. Assemblée nationale du Québec, Débats de l'Assemblée législative du Québec, Quatrième session, $25^{\mathrm{e}}$ législature, mardi le 14 décembre 1959, 53. Paul Sauvé tient des propos similaires en d'autres occasions: «Le gouvernement de la province de Québec ne renie pas le passé et il n’y a rien de surprenant à ce qu'il pose aujourd'hui la conclusion de l'œuvre qu'il a édifiée depuis quinze ans sous la direction de l'hon. Maurice Duplessis. Nous en faisons que continuer ce que nous avons entrepris en 1944 ». Henri Dutil, «La conclusion de l’œuvre de M. Duplessis, affirme le premier ministre Paul Sauvé», Le Soleil, 25 novembre 1959, 3 . 
On lui fait dire ce mot, ce vocable, ce marqueur de rupture temporelle. Le «Désormais... » en vient ainsi à symboliser toute la politique de Sauvé, d'où le fait que certains textes du Devoir le présentent ainsi, en novembre et en décembre 1959. Grâce à ce mot, la contradiction entre les paroles et les gestes de Paul Sauvé n'est plus. Comme le souligne Gérard Bouchard, le mythe - tout comme le mythistoire, avançons-nous - possède la faculté de résoudre ou de surmonter les contradictions ${ }^{107}$. C'est précisément ce qui se produit dans le cas du «Désormais... » de Paul Sauvé.

Ce mythistoire voile toutefois bien plus qu'il ne dévoile. S'il permet en effet d'articuler les deux termes de cette contradiction, il ne rend toutefois pas justice à la complexité de l'époque. Rappelons à cet effet les propos de Laurendeau, du 28 novembre 1959: il constate alors que Sauvé «ne dit plus "désormais...”"; «M. Sauvé, cette fois, n’a pas dit désormais. Là-dessus, le duplessisme continue ${ }^{108}$.» Le mythistoire aurait-il déjà épuisé toute sa charge symbolique, en l'espace de quelques jours à peine? Cela montre plutôt que, malgré ce mythistoire dont la fonction première est pourtant d'aplanir la contradiction, la contradiction chez Sauvé demeure entière, et ce, même chez André Laurendeau qui reste le premier promoteur de ce mythistoire. De fait, la contradiction est loin d'être résolue; l'éditorialiste du Devoir chantera de nouveau les louanges du premier ministre par la suite, du reste.

\section{CONCLUSION}

«Désormais...». Dans la littérature, du moment que Paul Sauvé en vient à prononcer le célèbre vocable, tout semble prendre forme. Par ce mot qu'il répète constamment, le successeur de Maurice Duplessis expie en quelque sorte les fautes, les péchés de son père politique, fondateur de l'Union nationale. En soi, le geste est formidable et d'une symbolique inouie. Au même titre qu'É.-Martin Meunier et Jean-Philippe Warren voient dans la Révolution tranquille une sortie religieuse de la religion ${ }^{109}$, nous voyons dans le «Désormais...» une sortie unioniste de la Grande Noirceur duplessiste qui prépare, voire amorce la Révolution tranquille. Pourrions-nous même dire que le "Désormais... " était trop beau pour être vrai? C’est une réalité avec laquelle il faudra, désormais, composer.

107. Gérard Bouchard, Raison et contradiction. Le mythe au secours de la pensée (Québec, Nota Bene, 2003), 129 p.

108. A. Laurendeau, "Où les "désormais" s'arrêtent», loc. cit., 4. Les italiques sont dans l'original.

109. É.-Martin Meunier et Jean-Philippe Warren, Sortir de la "Grande noirceur» : l'horizon "personnaliste» de la Révolution tranquille (Sillery, Septentrion, 2002), 207 p. 
Pourtant, nombreux ont été les chercheurs (historiens, politologues, sociologues et autres) à soutenir que Paul Sauvé a prononcé le "Désormais...». Par leurs travaux, ils ont contribué à solidifier les assises même du mythistoire, et ce, jusqu'à sa consécration en 2010 comme lieu de mémoire lors du cinquantième anniversaire de la Révolution tranquille. Ce faisant, le «Désormais...» est devenu une source, un témoignage de la rupture survenue au tournant des années 1960, avec la Révolution tranquille. Une source dont la plupart des analystes continuent de faire état, aujourd'hui.

Ironiquement, cette présence du «Désormais...» dans la mémoire est devenue un irritant pour ces mêmes chercheurs. C’est le cas d’Éric Bédard qui s'active, ces dernières années, à battre en brèche cette idée surfaite selon laquelle 1960 constitue l'an zéro du Québec moderne face à la Grande Noirceur ${ }^{110}$. C'est à cette fin qu'il propose dans son dernier ouvrage, L'Histoire du Québec pour les Nuls, d'élargir les bornes chronologiques de la Révolution tranquille pour en faire la «Reconquête tranquille» (1939-1967), laquelle englobe la période dite de la Grande Noirceur, pour mieux la dissoudre. Mais Éric Bédard est parti de la mémoire collective pour construire ce récit. À ce titre, il ne pouvait pas se permettre d'exclure le «Désormais...» de sa trame narrative, bien que cela lui nuise dans son entreprise; de son propre aveu, les lecteurs s'attendaient à l'y retrouver ${ }^{111}$. Aussi, a-t-il intégré le «Désormais... » à son récit, minant quelque peu son interprétation tout en accentuant la rupture entre la Grande Noirceur et la Révolution tranquille ${ }^{112}$.

Il faut dire que le «Désormais...» n’a pas toujours occupé cette place immuable dans la mémoire collective qu'on lui connaît aujourd'hui. Cela ne s'est pas fait du jour au lendemain, dès la mort de Paul Sauvé. En effet, lors de ses funérailles, de nombreuses personnalités politiques et médiatiques sont appelées à revenir sur son œuvre, dont André Laurendeau qui n'en glissera mot. Du reste, Pierre Laporte et lui signent quelques textes à cette occasion dans les pages du Devoir, mais ni l'un ni l'autre ne soulignent ce "mot en passe de devenir fameux ${ }^{113}$ ".

Quelques mois plus tard, tout juste avant le déclenchement des élections qui verront Jean Lesage l'emporter, Pierre Laporte fait paraître Le vrai visage de Duplessis. Comme le titre de l'ouvrage l’indique, Laporte prétend dévoiler

110. Voir Éric Bédard, Recours aux sources. Essais sur notre rapport au passé (Montréal, Boréal, 2011), 280 p.

111. Nous avons eu l'occasion d'en discuter avec Éric Bédard.

112. É. Bédard, L'Histoire du Québec..., op. cit., 205-263.

113. P. Laporte, " "Désormais"...", loc. cit., 1. 
l'ancien premier ministre sous son véritable jour. Pour ce faire, il revient notamment sur ses relations avec ses ministres, auxquels il consacre un chapitre. Le chapitre débute par ce mot d'esprit que lui aurait communiqué un sénateur québécois, selon lequel il y aurait trois sortes de ministres: "ceux qui sont assez intimes pour dire Oui, Maurice, ceux qui disent Oui, cheuf... et il y a Paul Sauvé114 !». L'occasion aurait été belle pour Laporte de souligner davantage l'exceptionnalisme de Sauvé au sein du cabinet, et ses distances vis-à-vis de Duplessis, en rappelant les «Désormais...» qu'il semait à profusion à l'automne 1959. Il ne l'a pas fait.

Enfin, lors de la campagne électorale de 1960, le successeur de Paul Sauvé, Antonio Barrette, fait campagne autour du slogan «Vers les sommets". La stratégie de l'Union nationale est d'associer Barrette à ses prédécesseurs ${ }^{15}$ : c'est ainsi qu'il apparaît sous une affiche électorale intitulée «Les trois grands». On peut y lire ce qui suit: «Ce qui frappe le plus la population du Québec, à la veille des prochaines élections provinciales, c'est la continuité de l'œuvre et des principes de base de l'Union nationale ${ }^{116}$.» De Maurice Duplessis à Antonio Barrette, en passant par Paul Sauvé, l'Union nationale fait alors le pari de la continuité - un pari qu'elle ne remportera pas, à vrai dire, mais cela importe peu. Il reste que ce pari est en tout point cohérent, conséquent avec les discours de Paul Sauvé qui, rappelons-le, martèle sans cesse qu'il poursuit «simplement le travail entrepris» par Maurice Duplessis ${ }^{117}$. Antonio Barrette continue dans la même lignée, le "Désormais... " n’ayant visiblement pas eu le temps de s'imposer au printemps 1960.

À partir de quels moments, autour de quels axes, de quels auteurs le «Désormais...» va-t-il s’imposer dans l'histoire et la mémoire du Québec? Il importe de s'intéresser de plus près aux différents usages du «Désormais...» dans la littérature et dans le politique, depuis le début des années 1960 jusqu'à nos jours. Ce sera l'objet de nos futures recherches. À terme, celles-ci permettront non seulement de mieux comprendre la construction symbolique d'un lieu de mémoire de la société québécoise, mais aussi comment s'écrit l'histoire du Québec depuis la Révolution tranquille.

114. Pierre Laporte, Le vrai visage de Duplessis (Montréal, Éditions de l’Homme, 1960), 65. Les italiques sont dans l'original.

115. Éric Bédard, «Le 22 juin 1960 - L'élection de Jean Lesage: un "changement de la vie”?», dans Pierre Graveline, dir., Dix journées qui ont fait le Québec (Montréal, VLB éditeur, 2013), 201.

116. Alain Lavigne, Duplessis, pièce manquante d'une légende. L'invention du marketing politique (Québec, Septentrion, 2012), 161.

117. Assemblée nationale du Québec, Débats de l'Assemblée législative du Québec, Quatrième session, $25^{\mathrm{e}}$ législature, mardi le 14 décembre 1959, 53. 